\title{
Phototoxicity revisited
}

\author{
As microscopy methods for studying biology in living samples advance and demand for them grows, assessment of \\ light damage caused by imaging becomes increasingly important.
}

T he ability to harness the power of light for imaging has had immeasurable impacts on our understanding of biology. Current microscopy methods can allow fast processes to be tracked in nearly real time and in super-resolution in whole cells, tissues, and organisms. However, achieving faster, higher-resolution, higher signal-to-noise, and deeper imaging often requires orders-of-magnitude greater light doses relative to those a biological specimen might experience naturally. It is now well understood that such light doses, and in some cases any light at all, can negatively affect sample fitness and viability.

This phenomenon, typically referred to as photodamage or phototoxicity, is a widely observed but rarely reported effect wherein researchers unwittingly change their sample simply by observing it. The extent to which these changes have affected the interpretation of imaging experiments is difficult to assess. However, as microscopy methods for studying living specimens are evolving rapidly and becoming increasingly in-demand, the time is right for developers and biologists to seek a more quantitative understanding of the effects of light on samples.

This is not to say that the problem of phototoxicity has not been examined in a systematic way. Numerous foundational studies have led to a few key observations. For example, it is well known that shorter light wavelengths are more damaging to cells, less light is always better, and different samples have different sensitivities to light. Other insights have also emerged, such as the fact that the nature and timing of illumination can affect outcomes, even in cases where the total light dose is constant.

Despite these important studies, predicting whether and when a specific imaging mode is appropriate for a given biological question or sample is extremely challenging. This is in part because of the huge diversity of biological questions being addressed by microscopy. Along these lines, a Correspondence in this issue from the Bewersdorf laboratory examines whether stimulated emission depletion (STED) nanoscopy is compatible with live imaging of fluorescently labeled cells grown in culture. This is a contentious issue, as STED light dose requirements are among the highest for super-resolution imaging.

In this work, the researchers used both short- and long-term assays to assess cell health, and found very little short-term stress on cells and good retention of longterm viability after STED imaging. This indicates at the very least that STED imaging does not mean certain death for cellsresults many may find astonishing. They also found that cell type matters and that the specific protein being labeled affects cells' susceptibility to photodamage. Imaging in buffers containing oxygen scavengers generally reduces short-term cell stress.

This study, although important for those using or considering STED, is far from comprehensive. For example, the researchers assayed only two cell types and used a single, far-red dye for all experiments, leaving it unclear whether their results will hold for imaging with other colors and other samples. In addition, there were fewer surviving cells in STED-imaged samples than in their unimaged counterparts, which indicates that phototoxicity cannot be completely ignored in STED nanoscopy. Still, systematic studies of this kind are needed to better understand the extent to which light is affecting biological observations.

For some time at Nature Methods we have been asking authors of manuscripts describing live-imaging methods to assay phototoxicity. We will continue to encourage such assessment, with a strong preference that it be included prior to peer review. As the field establishes standards for the measurement of phototoxicity, we envision that this will be a requirement for all manuscripts describing imaging of living samples.

Because phototoxicity depends strongly on the sample, question, and instrument, we will not require that a specific assay be done in any case; rather, the authors should apply one that matches the biological demonstration to which their method is targeted. For example, cell biologists using a new method to monitor secretion over short periods might choose to test for short-term stress after imaging, as well as to determine, using a non-imaging-based approach, whether secretion was affected by the imaging compared with that in dark controls. As another example, researchers conducting neuroimaging might choose to show that cell morphology and animal behavior are not affected by imaging over a relevant time scale.

As described in a Commentary on phototoxicity by Laissue et al., published in Nature Methods in 2017, researchers are encouraged to increase illumination until they observe phototoxic effects, and to then make sure to conduct their experiments with light well below that toxic level in cases where there is no obvious assay for stress or viability. We also strongly encourage developers of new optical setups to report the power density of light at the sample used to generate the images shown for publication, to facilitate cross-modality comparisons.

The benefits of this sort of assessment will be numerous. First and foremost, we hope to make it clearer to biologists and potential users the types of experiments for which methods and optical setups are and are not useful, and thereby reduce artifacts and improve discovery. These types of assays will also advance our collective understanding of the phenomenon of phototoxicity and raise awareness of its widespread nature. Finally, over time, standard assays for various sample types and microscopes will emerge to facilitate the comparison of methods.

Although it may be easy to focus on generating the most 'beautiful' images possible with a new method, it is also important for a method to deliver meaningful biological results. For live imaging, these goals may find themselves at odds, and gentler imaging should be the victor.

Published online: 1 October 2018 https://doi.org/10.1038/s41592-018-0170-4 